Artículo Original

\title{
Las vacunas de la malaria y su potencial aportación para el control y eliminación de la enfermedad
}

\author{
Laura Monzón Llamas ${ }^{1}$, Daniel Cobos Muñoz ${ }^{2}$, Paloma Merino Amador 3,* \\ 1 London School of Hygiene \& Tropical Medicine, SAMU, Swiss TPH, Universidad de las Palmas de Gran \\ Canaria Facultad de Ciencias de la Salud; ORCID id: https://orcid.org/0000-0002-5463-5744. \\ 2 Departamento de Epidemiología y Salud Pública del instituto Suizo de Medicina Tropical y Salud Pública \\ Basilea; ORCID id: https://orcid.org/0000-0002-5920-1304. \\ 3 Servicio de Microbiología Clínica del Hospital Clínico San Carlo de Madrid, Profesora Asociada del \\ Departamento de Medicina de la Facultad de Medicina de la UCM; ORCID id: https:/orcid.org/0000-0003- \\ 3524-9415 \\ * Autor correspondencia: palomeri@pdi.ucm.es \\ DOI: https://doi.org/10.37536/RIECS.2021.6.S1.248
}

Resumen: A pesar de que la malaria se puede prevenir y tratar, sigue ejerciendo efectos devastadores en la salud y el modo de vida de las personas a nivel mundial. La comunidad internacional lleva décadas realizando esfuerzos conjuntos para la eliminación del paludismo, lo que ha supuesto importantes cambios en las estrategias de salud pública adoptadas por los países en la lucha contra esta enfermedad. La vacunación es una de las estrategias fundamentales para el control de las enfermedades infecciosas, sin embargo, no siempre se puede lograr de manera sencilla. Por suerte la vacunología es uno de los campos donde más se ha avanzado en las últimas décadas y existen varios ensayos clínicos en desarrollo de los que se esperan buenos resultados. La disponibilidad de vacunas seguras que proporcionen una protección duradera contra la malaria puede suponer un importante avance en la lucha contra la enfermedad, y debe ser estudiada en el contexto de la aplicación de otras intervenciones efectivas, como son el uso de mosquiteros tratados con insecticida y el acceso a diagnóstico y tratamientos combinados con artemisinina.

Palabras Clave: Malaria, Vacunas, Control y eliminación de la malaria.

\begin{abstract}
Despite the fact that malaria can be prevented and treated, it still produces devastating effects on health and wellbeing of millions worldwide. Due to global, national and local efforts towards malaria control and elimination, the landscape of public health strategies in the endemic countries is changing rapidly. Malaria vaccines are being developed and has reached a stage where they have been taken to phase III and phase IV trials. Vaccination is an essential strategy for infectious disease control. However, the integration of new vaccines in an existing vaccination plan is not an easy endeavour. The availability of safe vaccines that produce long-lasting protection can be a considerable move in the fight against malaria worldwide. This should be studied in context where other effective public health interventions, such as long-lasting insecticide nets and access to rapid diagnosis and malaria combined treatment, are being applied.
\end{abstract}

Key words: Malaria, Vaccines, Malaria control and elimination. 


\section{Situación global}

A pesar de que la malaria se puede prevenir y tratar, sigue ejerciendo efectos devastadores en la salud y el modo de vida de las personas a nivel mundial. Se estima que los casos en el año 2019 fueron de 229 millones en todo el mundo. Plasmodium falciparum ha sido el parásito responsable de la mayoría de las muertes por malaria, debido a su diversidad y habilidad para esquivar las respuestas inmunológicas [1]. Actualmente existen 87 países endémicos, siendo la región africana la que reporta más del $90 \%$ de los casos y de las muertes por paludismo [2].

Según los resultados del estudio del GBD (Global Burden of Disease), realizado por IHME (Institute for Health Metrics and Evaluation), la mayor carga de mortalidad por malaria en los países subsaharianos se concentra en la franja de edad de niños menores de 5 años [3] (ver figura 1).

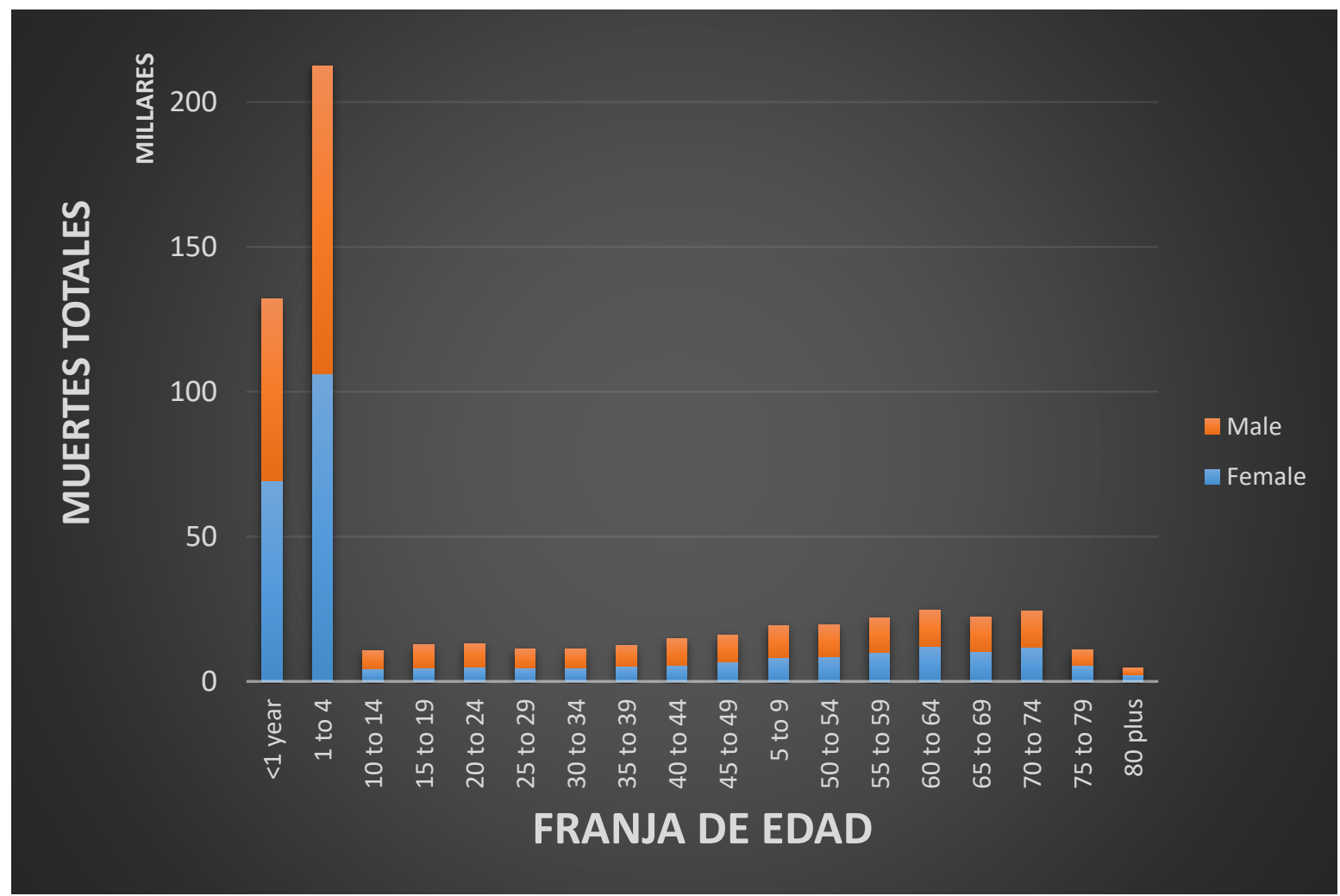

Figura 1 Distribución de muertes por malaria según franja de edad y sexo, en países sub-saharianos. Elaboración propia

En 2019, 32 países reportaron el 95\% de las muertes por malaria, encabezados por Nigeria que reportó el 23\% de las muertes por malaria (Ver figura 2). 


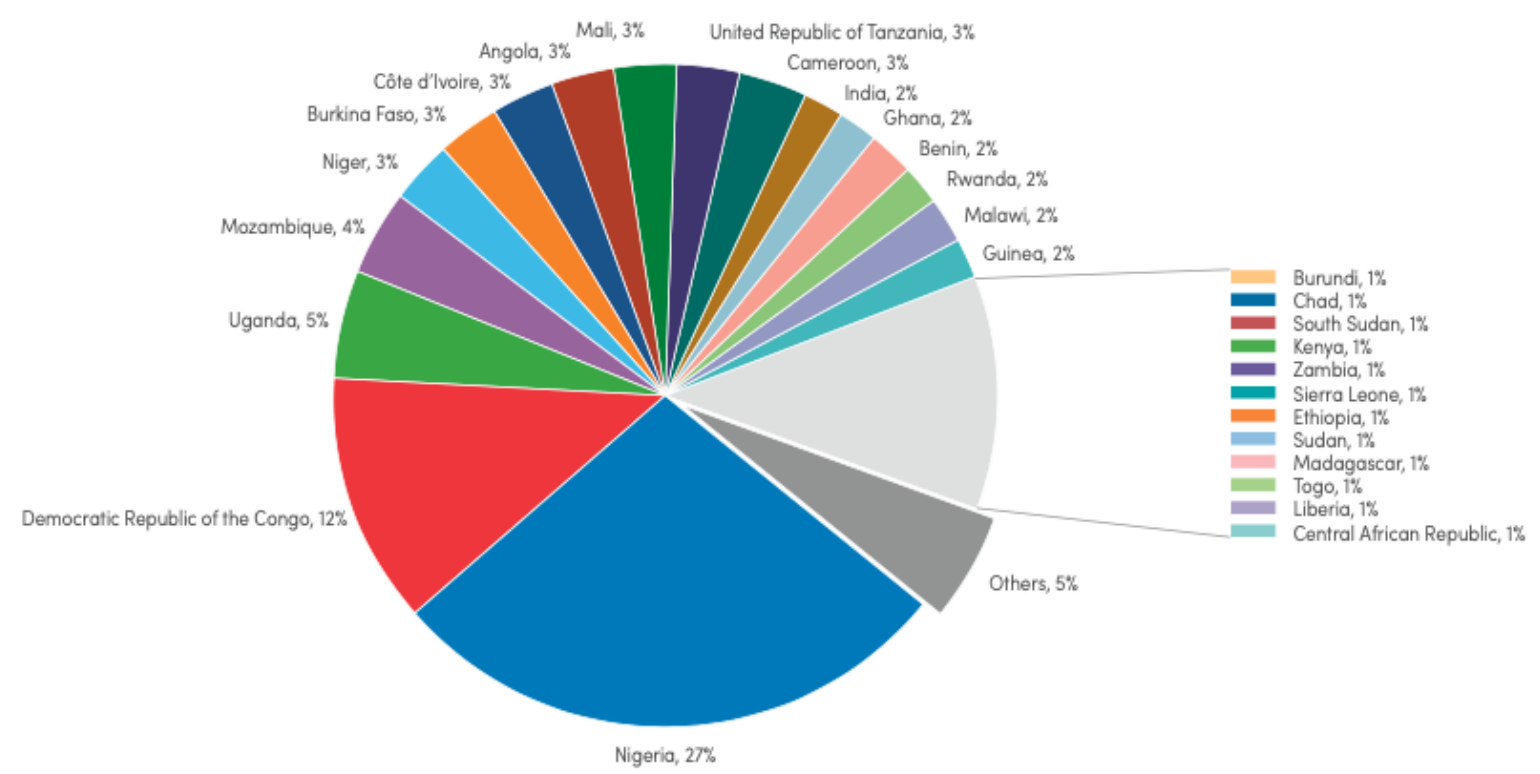

Figura 2 Porcentaje de muertes atribuibles por malaria en cada uno de los 32 países donde se produce el 95\% de las muertes por malaria (datos de 2019. Figura extraída del World Malaria Report 2020, Organización Mundial de la Salud)

Teniendo en cuenta las previsiones de crecimiento de la población mundial para el año 2030, así como el cambio en las condiciones medioambientales favorecidas por el cambio climático (que dificultan el control de vectores), se espera que el número de personas en riesgo de contraer la malaria aumente considerablemente. Esta circunstancia impondrá más exigencias a los sistemas nacionales de salud y a los presupuestos de los programas antimaláricos [4].

Además, en los países en los que la malaria ya supone una alta carga de morbi-mortalidad, existe una transición epidemiológica hacia las enfermedades no transmisibles [5]. Esto supone una carga extra para los sistemas de salud que también tienen que integrar el manejo de enfermedades crónicas [6], por lo que los recursos deben distribuirse y equipararse, quedando menos recursos disponibles para enfermedades transmisibles como el paludismo.

Consecuentemente la malaria se traduce en enormes pérdidas humanas y económicas para países y poblaciones que ya viven en entornos de constante vulnerabilidad y dificultad. Se hace evidente la necesidad de aumentar el número de personas que se benefician de intervenciones efectivas, así como de introducir nuevas intervenciones y en este caso una vacuna segura y costo efectiva que proporcione protección contra la malaria sería una importante aportación para las estrategias de control y eliminación de la enfermedad [1].

En base a recientes modelos matemáticos de proyección del impacto, en regiones con una endemicidad del 10-65\% de malaria (Plasmodium falciparum), la actual vacuna con estudio en fase III (RTS,S/AS01) evitaría una media de 93,9 casos clínicos y 394 muertes por cada cien mil niños completamente vacunados (con tres dosis). Para implementar la vacunación deben considerarse factores como la carga de la enfermedad, el coste-efectividad de la intervención y la cobertura de otras intervenciones destinadas al control y eliminación de la malaria [7]. Por tanto, la vacunación debe ser una intervención más a integrar en las estrategias de control y eliminación de la malaria, según el contexto de cada entorno y sistema de salud.

De este modo, así como actualmente se utiliza la "teoría del queso suizo" para explicar la relevancia de aplicar diferentes medidas en el control la pandemia producida por el virus SARSCOV-2, podríamos aplicar la misma teoría para explicar la relevancia de la aplicación de diferentes intervenciones para controlar la malaria en países donde la enfermedad es endémica. La teoría explica como la aplicación de múltiples medidas de protección, representadas por las rebanadas de queso, impiden la transmisión de la enfermedad. Ninguna rebanada es perfecta, ya que todas tienen agujeros, así que cuando los agujeros coinciden de forma alineada a lo largo de las rebanadas 
aumenta el riesgo de infección. Sin embargo, varias rebanadas combinadas reducen significativamente el riesgo total. La vacunación añadiría una capa más de protección [8].

\section{Esfuerzos y desafíos para el control y la eliminación de la malaria}

Hace más de cincuenta años que la comunidad internacional viene realizando esfuerzos conjuntos para la eliminación del paludismo. Esta circunstancia ha supuesto importantes cambios en las estrategias adoptadas por los países endémicos y en las prioridades de la comunidad científica y sus financiadores. Los objetivos de los programas de la nueva estrategia para el control de la enfermedad, oscilan entre reducir la carga y mantenerla a un nivel razonablemente bajo, eliminar la enfermedad en determinadas áreas geográficas y finalmente, erradicarla [9].

Desde el año 2000, estos cambios de estrategia se han traducido en una importante mejora en las coberturas de intervenciones de salud pública en África subsahariana. Se estima que estas intervenciones han evitado 663 millones de casos desde el año 2000, y concretamente, el uso de mosquiteras tratados con insecticida ha contribuido al 68\% de los casos evitados [10].

Otras intervenciones que han contribuido a esta reducción en el número de casos son el uso de insecticidas para el control de vectores en zonas donde habita, y el acceso a diagnóstico y tratamiento de las poblaciones afectadas.

Las terapias antimaláricas han demostrado reducir la morbilidad y mortalidad de la malaria al acabar con la infección y frenar la transmisión a través de la disminución del reservorio del parásito. Diversos medicamentos han permitido avanzar en la lucha contra el paludismo, desde la tradicionalmente usada cloroquina, hasta tratamientos más recientes, como la autovacuonaproguanil, que han ido desarrollando resistencias a lo largo del tiempo y uso. Actualmente, la terapia más efectiva se basa en la combinación de artemisinina con otro fármaco asociado para demorar la aparición de resistencias [11].

La evidencia reciente también ha puesto de manifiesto la resistencia desarrollada a los cuatro insecticidas más comúnmente utilizados en la lucha contra la malaria: piretroides, organoclorados, carbamatos y organofosforados. Esta resistencia se ha extendido en la mayor parte de vectores de la malaria en las regiones de África, América, Sureste asiático, Mediterráneo oriental y Pacífico occidental [12].

La resistencia a los medicamentos antimaláricos supone una traba para el control de la enfermedad. Aunque la prevalencia de la resistencia a la cloroquina ha descendido, esta droga se encuentra en desuso. En principio, la terapia combinada con artemisinina actualmente no presenta resistencias significativas en África. Sin embargo, en Asia, la resistencia a esta terapia ya supone un reto para el tratamiento del paludismo, y si esta situación se replicara en África supondría unas consecuencias devastadoras en la lucha global contra la malaria [13].

Por otra parte, la exposición continua a picaduras de mosquitos infectados da lugar a que se desencadene una reacción inmunitaria contra el parásito y contra la enfermedad. El nivel de esta respuesta inmunitaria viene determinado por la intensidad de la transmisión y la epidemiología de la enfermedad. Se ha demostrado que la prevalencia submicroscópica del parásito es mayor en zonas de baja transmisión y es bastante común en niños, probablemente como resultado de una respuesta inmune menos robusta que no tiene tiempo suficiente para producir una inmunidad protectora. Además, las infecciones asintomáticas pueden persistir durante meses. La presencia de infecciones submicroscópicas y asintomáticas suponen un desafío significativo para el control y eliminación de la malaria, ya que perpetúan la transmisión [1]. 


\section{Aportaciones de la vacuna}

Recientes avances en vacunología, basados en protección inmunológica correlativa, secuenciación linfocítica y diseño estructural de inmunógenos, proporcionan una perspectiva integral para la identificación y el diseño de vacunas altamente eficaces para la malaria [1].

Por otra parte, las vacunas que actualmente se encuentran en desarrollo están enfocadas en todas y cada una de las fases del ciclo del parásito. Sin embrago, las que poseen una mayor eficacia demostrada actúan en la fase pre-eritrocítica a través de la inoculación de esporozoítos atenuados o vacunas de subunidad [14].

La disponibilidad de vacunas seguras que proporcionen una protección duradera contra la malaria supondría un importante avance en la lucha contra la enfermedad, y debería ser estudiada en el contexto de la aplicación de otras intervenciones efectivas, como son el uso de mosquiteras tratados con insecticida y el acceso a diagnóstico y tratamientos combinados con artemisinina.

\section{Vacunas en desarrollo}

Actualmente existen dos tipos de vacunas contra la malaria: vacunas de subunidad y vacunas de esporozoítos atenuados. La más desarrollada es la vacuna de subunidad RTS,S/AS01E, con una eficacia demostrada limitada pero de más fácil aplicación y conservación. Se está investigando otra vacuna, también de subunidad que posee mayores expectativas de eficacia, la R21.

Las vacunas de esporozoítos atenuados actualmente disponen de una mayor eficacia demostrada, pero resulta de difícil aplicación en zonas endémicas debido a sus condiciones de conservación y administración (intravenosa y con dosis frecuentes).

A continuación, presentamos cada una de ellas.

\subsection{Vacunas de subunidad}

\subsubsection{Vacuna RTS,S/AS01E}

La vacuna RTS, S actúa sobre la proteína circumsporozoíto en la fase pre-eritrocítica de la infección por Plasmodium falciparum, e induce una protección inmunológica mediada por anticuerpos anti-circumsporozoíto. Administrada con el adyuvante AS02A ha demostrado una eficacia del 30\% en niños de entre 1 y 4 años.

Investigaciones más recientes con un adyuvante más inmunogénico (AS01E) han demostrado una mayor eficacia en niños entre 5 y 17 meses de edad [15]. Durante los primeros 18 meses de seguimiento tras la administración de 3 dosis de la vacuna, se demostró un $46 \%$ de eficacia contra la infección en niños entre 5 y 17 meses de edad, y una eficacia de $27 \%$ en lactantes de 6 a 12 semanas de edad [16]. Dicha eficacia disminuyó durante los 38-48 meses de seguimiento posteriores a 28,3\% y $18,3 \%$ respectivamente [17]. El estudio no demostró correlación entre el nivel de anticuerpos anticircumsporozoíto en sangre y el nivel de protección frente a la infección.

Entre 2009 y 2014 se llevó a cabo el ensayo clínico en fase 3 en niños entre 5-17 meses en siete países africanos. Se demostró que se prevenían hasta el 39\% de los casos de malaria, y el 29\% de los casos de malaria grave [18].

En el año 2015, tras consideraciones respecto del impacto potencial, la factibilidad de la implementación de la vacunación y la relación coste-efectividad de la vacuna, la OMS hizo pública la recomendación de desarrollar más estudios para la implementación de la vacunación, con el objetivo de rellenar las lagunas existentes. La eficacia demostrada en el grupo de niños entre 5 y 17 meses se consideraba todavía muy baja para justificar la implementación de la vacunación [19]. En este contexto, en 2019 la OMS inició un estudio aleatorio en fase IV en Malawi, Mozambique y Ghana [20]. 


\subsubsection{Vacuna R21}

En el año 2016 se publicaron los resultados del estudio de una vacuna más inmunogénica que también actúa sobre la proteína circumsporozoíto (PCS). Particularmente, está desarrollada a partir de antígenos de superficie del virus de la hepatitis B, lo que lleva a una mayor proporción de PCS en la composición de la vacuna.

La vacuna ha sido probada en ratones y ha demostrado inmunogenicidad a dosis muy bajas. Administrada con adyuvantes específicos genera una respuesta protectora contra potenciales mutaciones [14].

\subsection{Vacunas de parásitos de la malaria atenuados}

Este tipo de vacunas maximiza el número de antígenos presentes por lo que limita el impacto de la diversidad antigénica en la eficacia de la vacuna [21].

\subsubsection{Parásitos atenuados químicamente in vivo}

Diversos estudios han demostrado que la administración de tratamiento antimalárico tras una infección primaria produce protección frente a infecciones secundarias, reduciendo la parasitemia y severidad de la enfermedad. Este régimen de infección seguido de tratamiento representa la atenuación química in vivo del parásito [21].

Se han llevado a cabo estrategias de inmunización con diferentes antimaláricos como la cloroquina o la mefloquina, en ratones y en humanos. Tras la inmunización se han desarrollado anticuerpos contra los esporozoítos, los parásitos en sangre y las proteínas recombinantes de los parásitos. También ha aumentado la producción de citoquinas en respuesta a la infección por Plasmodium spp en sangre [22]. La protección inducida es de larga duración, manteniéndose hasta 28 meses tras la infección primaria [23].

\subsubsection{Parásitos atenuados químicamente in vitro}

Este método consiste en atenuar químicamente esporozoítos in vitro que posteriormente se inoculan en sangre, produciendo protección frente a futuras infecciones. La inmunización con parásitos atenuados induce también protección contra cepas y especies heterólogas [24].

\section{Desafíos de la vacuna}

El principal desafío al que se enfrenta la vacuna contra la malaria es la integración de la vacunación en el sistema de salud, como intervención integrada en la estrategia para el control y eliminación de la malaria. La necesidad de disponer de sistemas de salud resilientes, con capacidad para adaptarse a nuevas dificultades, como son nuevas epidemias/pandemias, desastres naturales, nuevas resistencias a los tratamientos e insecticidas disponibles, etc...se pone de manifiesto también a la hora de integrar nuevas intervenciones.

La vacunación contra la malaria debería poder integrarse en el calendario vacunación que ya está en funcionamiento ya que la adherencia a los programas de vacunación supone actualmente un reto en zonas endémicas [25] en las que la exposición a numerosas enfermedades infecciosas está a la orden del día.

En las vacunas de subunidad la eficacia demostrada es reducida y se limita al rango de edad entre 5 y 7 meses de edad, para los adultos estas cifran disminuyen a $34 \%$ de eficacia en estudios preliminares [26]. Además, según la evidencia disponible no parece que la protección se prolongue en el tiempo, por lo que los niños vacunados estarían de nuevo expuestos a la malaria desde muy corta edad. No obstante, según datos de IHME, dada la carga de mortalidad en este grupo de edad [3], de ser efectiva la vacuna supondría un gran impacto para la salud de estos países.

Estudios recientes basados en elaboración de modelos y proyecciones, sugieren que, en lugares de alta transmisión, las estrategias actuales para el control y eliminación de la malaria representan la intervención más eficiente. No obstante, incluir estrategias de vacunación en entornos específicos de baja transmisión (incluso con coberturas bajas de vacunación) sería más eficiente que una vacunación 
masiva de niños a nivel de país. La viabilidad y el coste de una vacunación masiva a este nivel resulta todavía bastante incierta y precisa de mayor evidencia [27].

Por otra parte, la administración de vacunas atenuadas in vivo requeriría múltiples ciclos de infección y administración de tratamiento controlados, lo que implicaría un nivel de dificultad considerable en la implementación de la vacunación en zonas endémicas. Hay también ciertos desafíos para el uso de vacunas atenuadas in vitro, como son el uso de sangre humana y productos sanguíneos, la posibilidad de inocular parásitos poco atenuados y la dificultad de almacenamiento e implementación de la vacunación (estas vacunas requieren de crioconservación). Sin embrago, nuevas estrategias para la crioconservación y administración de tratamientos podrían reducir estas barreras [21].

Los esfuerzos deben encaminarse a mejorar las capacidades, tanto en recursos humanos como en infraestructuras, para monitorear y gestionar de forma eficiente las resistencias a los insecticidas, así como para disponer de nuevos productos (insecticidas, tratamientos y vacunas) adecuados y accesibles, para el control de la malaria [28]. La forma de asegurar una correcta implementación de estrategias para el control y eliminación de la malaria es alineando esfuerzos y asignando los recursos necesarios a los sistemas de salud.

\section{Conclusión}

La vacunación es una de las estrategias fundamentales para el control de las enfermedades infecciosas, sin embargo, no siempre se puede lograr de manera sencilla. En el caso de las enfermedades parasitarias y en concreto en la malaria, este método resulta más complejo por las características microbiológicas de Plasmodium spp. Por suerte la vacunología es uno de los campos donde más se ha avanzado en las últimas décadas y existen varios ensayos clínicos en desarrollo y se esperan buenos resultados. En el año 2019, la OMS recomendó la introducción de la vacuna RTS, S / AS01 (RTS, S) en tres países africanos donde se está realizando un programa piloto y ya se han administrados más de un millón de dosis [24].

\section{Referencias bibliográficas}

1. Frimpong A, Asamoah Kusi K, Fokuo Ofori M, Ndifon W. Novel Strategies for Malaria Vaccine

2. Design, Front. Immunol 2018; 9:2769.

3. WHO, World Malaria Report 2020: 20 Years of Global Progress and Challenges (Geneva: World Health Organization; 2020. Licence: CC BY-NC-SA 3.0 IGO., 2020).

4. Institute for Health Metrics and Evaluation, 'GBD Compare' (IHME, University of Washington, 2015) $<$ http://www.healthdata.org/gbd/2019>.

5. Estrategia Técnica Mundial Contra La Malaria 2016-2030 (Organización Mundial de la Salud, 2015).

6. Atiim GA, Elliot SJ. The Global Epidemiologic Transition: Noncommunicable Diseases and Emerging Health Risk of Allergic Disease in Sub-Saharan Africa, Health Educ Behav 2016; 43(1 Suppl):37S-55S

7. Stower H. A Disease Transition in Sub-Saharan Africa, Nat Med., 25(11):1647

8. Penny MA et al. Public Health Impact and Cost-Effectiveness of the RTS,S/AS01 Malaria Vaccine: A Systematic Comparison of Predictions from Four Mathematical Models, The Lancet 2016;387:367-75.

9. Roberts S. The Swiss Cheese Model of Pandemic Defense: It's Not Edible, but It Can Save Lives. The Virologist Ian Mackay Explains How, The New York Times, Dec. 5, 2020.

10. WHO, Global Malaria Control and Elimination: Report of a Technical Review (Geneva, Switzerland, 17 January 2008).

11. Bhatt S. Weiss DJ, Cameron E, Bisanzio D, Mappin B, Dalrymple U, et al. The Effect of Malaria Control on Plasmodium falciparum in Africa between 2000 and 2015 Nature 2015; 526:207.

12. WHO, Global Malaria Control and Elimination: Report of a Technical Review. 2020.

13. WHO, 'Malaria: Insecticide Resistance' (WHO: web page, 2020).

14. Philip JR. Antimalarial Drug Resistance in Africa: The Calm before the Storm? The Lancet 2019; 19.10.

15. Collins KA., Snaith R, Cottingham MG, Gilbert SC, Hill AVS. Enhancing Protective Immunity to Malaria with a Highly Immunogenic Virus-like Particle Vaccine, Scientific Reports 2017; 7:46621. 
16. Bejon P et al, Efficacy of RTS,S/AS01E Vaccine against Malaria in Children 5 to 17 Months of Age, New England J Med 2008; 359.24: 2521-2532.

17. The RTS,S Clinical Trials Partnership. Efficacy and Safety of the RTS,S/AS01 Malaria Vaccine during 18 Months after Vaccination: A Phase 3 Randomized, Controlled Trial in Children and Young Infants at 11 African Sites, 2014, PLoS Medicine.

18. Greenwood BM. Efficacy and Safety of RTS,S/AS01 Malaria Vaccine with or without a Booster Dose in Infants and Children in Africa: Final Results of a Phase 3, Individually Randomised, Controlled Trial, Lancet 2015386(9988): 31-45.

19. The RTS,S Clinical Trials Partnership, 'First Results of Phase 3 Trial of RTS,S/AS01 Malaria Vaccine in African Children', N Engl J Med 2011; 365:1863-1875.

20. Malaria Vaccine: WHO Position Paper' (World Health Organisation No 4, 33-52 (2016).

21. World Health Organization, First Malaria Vaccine in Africa: A Potential New Tool for Child Health and Improved Malaria Control.

22. Raja AI, Stanisic DI, Good MF. Chemical Attenuation in the Development of a Whole-Organism Malaria Vaccine, Infect Immun, 2017; 85:e00062-17.

23. Roestenberg M. McCall M, Hopman J, Wiersma J, Luty AJF, van Gemert GJ, van de Vegte-Bolmer M, van Schaijk B, Teelen K, Arens T, Spaarman L, de Mast Q, Roeffen W, Snounou G, Renia L, van der Ven A, Hermsen CC, Sauerwein R. Protection against a Malaria Challenge by Sporozoite Inoculation N Engl J Med, $2009 ; 361: 468-477$.

24. Roestenberg M, Teirlinck AC, McCall MBB, Teelen K, Makamdop KN, Wi- ersma J, Arens T, Beckers P, van Gemert G, van de Vegte-Bolmer M, van der Ven AJAM, Luty AJF, Hermsen CC, Sauerwein RW. LongTerm Protection against Malaria after Experimental Sporozoite Inoculation: An Open-Label Follow-up Study, Lancet, 2011; 377:1770 -1776.

25. Good MF. Reiman JM, Rodriguez IB, Ito K, Yanow SK, El-Deeb IM, Batzloff MR, Stanisic DI, Engwerda C, Spithill T, Hoffman SL, Moses L, McPhun V. Cross-Species Malaria Immunity Induced by Chemically Attenuated Parasites, J Clin Invest, 2013; 66634.

26. Kazungu JS, Adetifa IMO. Crude Childhood Vaccination Coverage in West Africa: Trends and Predictors of Completeness, Wellcome Open Res 2017; 2: 12.

27. Bejon P et al. Stable and Unstable Malaria Hotspots in Longitudinal Cohort Studies in Kenya. Plos Medicine, 2010.

28. Smith T, Ross A, Maire N, Chitnis N, Studer A, Hardy D, Brooks A, Penny M, Tanner M . Ensemble Modeling of the Likely Public Health Impact of a Pre-Erythrocytic Malaria Vaccine, PLoS Med 2012 Jan;9(1):e1001157.

29. Mnzava A, Knox T, Temu EA, Trett A. Implementation of the Global Plan for Insecticide Resistance Management in Malaria Vectors: Progress, Challenges and the Way Forward, Malaria Journal 201; 14(1):173.

(C) 2021 por los autores; Esta obra está sujeta a la licencia de Reconocimiento 4.0 Internacional de Creative Commons. Para ver una copia de esta licencia, visite http://creativecommons.org/licenses/by-nc-nd/4.0/. 